\title{
Dilaton from tractor and matter field from twistor
}

\section{J. François}

Service de Physique de l'Univers, Champs et Gravitation, Université de Mons - UMONS, 20 Place du Parc, B-7000 Mons, Belgium

E-mail: jordan.francois@umons.ac.be

ABSTRACT: Conformal tractors and twistors can be obtained via gauge reduction of the conformal Cartan geometry, thanks to the dressing field method. Perhaps surprisingly, it is possible to reduce the gauge symmetry further still. The Weyl symmetry can indeed be erased thanks to the tractor field, from which a dilaton is extracted. This suggests an alternative to the Weyl or conformal spontaneous symmetry breaking (SSB) that some authors proposed as improvement of the Standard Model or of inflationary cosmology, but also raises doubts as to the physical significance of such symmetries. Here, after gauge reduction via dressing, only the Lorentz gauge symmetry remains physically relevant, and the twistor field becomes - for all practical purposes - a Dirac spinor field. In a simple illustrative toy model, the latter acquires a mass through Lorentz SSB due to the VEV of the Weyl-invariant tractor field.

Keywords: Conformal and W Symmetry, Differential and Algebraic Geometry, Gauge Symmetry, Spontaneous Symmetry Breaking

ARXIV EPRINT: 1810.07976 


\section{Contents}

1 Introduction 1

2 Reduction of gauge symmetries via dressing 2

2.1 Invariant composite fields 2

2.2 Residual gauge symmetries 4

3 Tractors and twistors from gauge reduction of the conformal Cartan geometry

$\begin{array}{lll}4 & \text { A dressing field for the Weyl gauge symmetry } & 8\end{array}$

5 Discussion $\quad 11$

$\begin{array}{ll}\text { A Dressing fields and Weyl structures } & 12\end{array}$

\section{Introduction}

Conformal and Weyl (or local scale) symmetries do not only elicit interest in exotic and/or low dimensional contexts such as string theory - where the world-sheet of strings is required to have exact Weyl symmetry — or in the literature related to AdS/CFT holography - where a gauge theory in a bulk is dual to a conformal field theory on the boundary. They are also put forward as relevant for plain 4-dimensional physics, though usually thought of as spontaneously broken by a so-called dilaton field. For example, the spontaneous breaking of the conformal $\mathrm{SO}(2,4)$ symmetry down to a $\mathrm{SO}(1,4)$ deSitter symmetry has been advocated as an alternative to cosmological inflation and as an explication of the near scale invariance of the CMB perturbations spectrum [1]. Weyl SSB as been proposed as a way to improve (or drive) inflationary cosmology, to stabilize the Standard Model (SM) and to give mass to all of its fields [2]. It has also been suggested that it may be the key to an improved SM where all parameters are computable, and to the solution of both the black hole information and the firewall problems [3].

Model building in 4D can take advantage of conformal differential geometric frameworks such as Penrose's Twistor theory, ${ }^{1}$ or the conformal Tractor calculus [6, 7]. Both deal only with Weyl gauge symmetry. The most general differential geometric framework to take on the full conformal symmetry is the conformal Cartan geometry [8], to which both twistor and tractor conformal calculi are closely related [9]. We intend to show how,

\footnotetext{
${ }^{1}$ Which was intended originally as a framework for all of physics, and short of that objective it is nevertheless of great conceptual and technical relevance in advanced topics such as string theory [4] and, to a lesser extent, loop quantum gravity [5].
} 
within this differential geometric setup, one can get ride of the Weyl gauge symmetry without SSB, but rather in a non-dynamical way via the dressing field method (DFM), thereby questioning its physical meaning. We notice the close relation with Stora's idea about the Wess-Zumino functional as expounded in [10] (appendix A).

The paper is organized as follows. Section 2 is a nutshell presentation of the dressing field method, where the main theorems regarding the gauge symmetry reduction and the residual gauge symmetries are stated in the form of propositions. Section 3 describes synthetically how the tractor and twistor bundles are obtained from gauge reduction of the conformal Cartan reduction via dressing. Given these prerequisites, section 4 shows how a dilaton is extracted from the tractor field and used to built a dressing field that allows to non-dynamically erase the Weyl gauge symmetry, so that only the residual Lorentz gauge symmetry seems physically relevant. In particular, one obtains a Weyl-invariant twistor that is identified with a Dirac spinor. This geometric construction is illustrated via a toy model in which the tractor fields, embedded in a potential, plays the same role as the scalar field in the electroweak model, and in which the invariant twistor is endowed with a mass through spontaneous breaking of the residual Lorentz symmetry. Of course this model is put forward merely to invite the reader to ponder the symmetry reduction mechanism presented. Finally, in section 5 we discuss the import of our main result concerning the non-dynamical reduction of Weyl symmetry, and in particular argue that (independently of our toy model) our geometric analysis support a claim made by Jackiw and Pi a few years ago [11] according to which the Weyl symmetry of — at the time - recently proposed cosmological models is what they dubbed 'fake'. We indicate that their work, and ours, can be situated in a broader context within philosophy of physics regarding the distinction between artificial and substantial gauge symmetries.

\section{Reduction of gauge symmetries via dressing}

The dressing field method is a technically rather simple and nonetheless conceptually powerful tool allowing to deal with gauge symmetries so as to reveal the physical degrees of freedom (d.o.f) of a theory in a way that differs markedly from both gauge fixing or spontaneous symmetry breaking mechanisms. One can consult [12] p.377 for a review with some interesting applications, and [13] for an assessment of its implications regarding philosophy of physics. This section is a nutshell presentation of the method and is the occasion of fixing some notation. We refer to [14] for the proofs of all the following assertions.

\subsection{Invariant composite fields}

The main purpose of the DFM is to produce gauge-invariant composite fields that can adequately represents the physical d.o.f of a gauge theory. It is better formulated in the language of fiber bundle differential geometry. So let us recall what is the geometric setup of a gauge theory.

The main object if a principal fiber bundle $\mathcal{P} \stackrel{\pi}{\rightarrow} \mathcal{M}$ over an $m$-dimensional spacetime $\mathcal{M}$ with structure Lie group $H$, also noted $\mathcal{P}(\mathcal{M}, H)$. The right action of $H$ on $\mathcal{P}$ is $R_{h} p=p h, \forall p \in \mathcal{P}$. The bundle is endowed with a h-valued (Ehresmann) connection 
1-form $\omega \in \Lambda^{1}(\mathcal{P}, \mathfrak{h})$, with curvature 2-form $\Omega \in \Lambda^{2}(\mathcal{P}, \mathfrak{h})$ given by Cartan's structure equation $\Omega=d \omega+\frac{1}{2}[\omega, \omega]$. For any choice of trivializing section $\sigma: \mathcal{U} \subset \mathcal{M} \rightarrow \mathcal{P}$ over an open subset of $\mathcal{M}$, one obtains by pullback the local representatives $A=\sigma^{*} \omega \in \Lambda^{1}(\mathcal{U}, \mathfrak{h})$ and $F=\sigma^{*} \Omega \in \Lambda^{2}(\mathcal{U}, \mathfrak{h})$ which are respectively the Yang-Mills gauge potential and its associated field-strength.

Given any representations $(\rho, V)$ for $H$ one built the associated bundle $E=\mathcal{P} \times{ }_{\rho} V$ over $\mathcal{M}$ whose sections $\Gamma(E)$ are in one to one correspondence with $V$-valued $H$-equivariant maps $\varphi: \mathcal{P} \rightarrow V$, i.e satisfying $R_{h}^{*} \varphi=\rho(h)^{-1} \varphi$. These represent matter fields. The connection form induces a covariant derivative on this space of maps/sections, $D \varphi=$ $d \varphi+\rho_{*}(\omega) \varphi$ - where the pushforward $\rho_{*}$ is the representation map of $\mathfrak{h}$ on $V$ - which represents the minimal coupling between the gauge potential and matter fields.

The diffeomorphisms of $\mathcal{P}$ that preserve its fibration form the group of vertical auto-

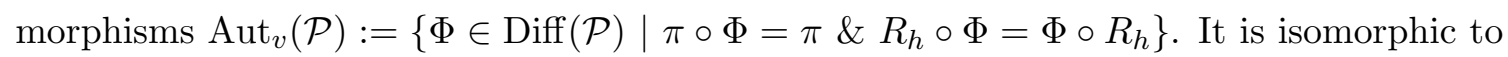
the gauge group $\mathcal{H}:=\left\{\gamma: \mathcal{P} \rightarrow H \mid R_{h}^{*} \gamma=h^{-1} \gamma h\right\}$, the isomorphism being $\Phi(p)=p \gamma(p)$. The gauge transformations of the basic field variables are then defined as,

$$
\begin{aligned}
& \omega^{\gamma}:=\Phi^{*} \omega=\gamma^{-1} \omega \gamma+\gamma^{-1} d \gamma, \quad \quad \Omega^{\gamma}:=\Phi^{*} \Omega=\gamma^{-1} \Omega \gamma, \\
& \varphi^{\gamma}:=\Phi^{*} \varphi=\rho\left(\gamma^{-1}\right) \varphi, \quad \text { and } \quad\left(D \varphi^{\gamma}\right):=\Phi^{*} D \varphi=\rho\left(\gamma^{-1}\right) D \varphi=D^{\gamma} \omega^{\gamma} \text {. }
\end{aligned}
$$

A physical gauge theory is given by an action functional $\mathcal{S}=\int_{\mathcal{M}} L$, with $L=L(A, \varphi)$ a Lagrangian m-(pseudo) form on $\mathcal{M}$ which is required to be gauge-invariant $-L\left(A^{\gamma}, \varphi^{\gamma}\right)=$ $L(A, \varphi)$ - or pseudo-invariant, i.e invariant up to an exact form - $L\left(A^{\gamma}, \varphi^{\gamma}\right)=L(A, \varphi)+$ $d \alpha(A, \varphi, \gamma)$

Given this geometric and gauge theoretic setup, the essential proposition of the DFM is the following.

Proposition 1. Let $K$ and $G$ be subgroups of $H$ such that (s.t) $K \subseteq G \subseteq H$. Note $\mathcal{K} \subseteq \mathcal{H}$ the gauge subgroup associated to $K$. Suppose there exists a map $u: \mathcal{P} \rightarrow G$ defined by its $K$-equivariance: $R_{k}^{*} u=k^{-1} u, \forall k \in K$, or equivalently by its $\mathcal{K}$-gauge transformation: $u^{\gamma}=\gamma^{-1} u, \forall \gamma \in \mathcal{K}$. Such a map, that we call a dressing field, allows to define the composite fields

$$
\begin{aligned}
& \omega^{u}:=u^{-1} \omega u+u^{-1} d u, \quad \quad \Omega^{u}=u^{-1} \Omega u=d \omega^{u}+\frac{1}{2}\left[\omega^{u}, \omega^{u}\right], \\
& \varphi^{u}:=\rho\left(u^{-1}\right) \varphi \quad \text { and } \quad D^{u} \varphi^{u}=\rho\left(u^{-1}\right) D \varphi=d \varphi^{u}+\rho_{*}\left(\omega^{u}\right) \varphi^{u} \text {, }
\end{aligned}
$$

which are $\mathcal{K}$-invariant, $K$-horizontal and therefore project (live on) the quotient subbundle $\mathcal{P} / K \subset \mathcal{P}$.

The first thing to make clear is that despite the formal resemblance, (2.2) must not be confused with (2.1). To perform a dressing operation is not to perform a gauge transformation, a composite field is not a gauge transform of a gauge variable. This is obvious if one is attentive to the fact that a dressing field, given its defining equivariance property, cannot be an element of the gauge group $(\mathcal{H})$ or one of its subgroup $(\mathcal{K})$. As a matter of fact, the composite fields (2.2) do not even belong to the original space of gauge variables. 
For example, $\omega^{u}$ has trivial $K$-equivariance and is $K$-horizontal, which implies that it is no longer a connection on $\mathcal{P}$. In particular this means that to perform a dressing is certainly not equivalent to fixing a gauge, i.e to select a single representative in the gauge orbit of any one gauge variable.

Locally, on $\mathcal{U} \subset \mathcal{M}$, keeping $u$ to denote the local dressing field (instead of $\sigma^{*} u$ ) and considering $\varphi$ as a section (not a map on $\mathcal{P}$ ), the local $\mathcal{K}$-invariant composite fields can be defined likewise: $A^{u}=u^{-1} A u+u^{-1} d u$ and $\varphi^{u}=\rho\left(u^{-1}\right) \varphi$. So if a $\mathcal{H}$-gauge theory is a priori given by $L(A, \varphi)$, but a (local $\left.{ }^{2}\right) \mathcal{K}$-dressing field exists, then - using the invariance of the Lagrangian which holds as a formal property - one can rewrite the theory in terms of $\mathcal{K}$-invariant variables as $L(A, \varphi)=L\left(A^{u}, \varphi^{u}\right)$. This shows that our theory is actually a $\mathcal{H} / \mathcal{K}$-gauge theory. Part of the gauge symmetry was spurious and could be eliminated by a change of field variables.

\subsection{Residual gauge symmetries}

The next interesting question concerns the residual gauge symmetries exhibited by the composite fields. For this question to even make immediate sense it is necessary to assume that $K$ is not merely a subgroup but a normal subgroup of $H, K \triangleleft H$, so that the quotient $H / K$ is a group in its own right, that we note $J$ for convenience. The quotient subbundle $\mathcal{P} / K$ is then a $J$-principal bundle $\mathcal{P}^{\prime}(\mathcal{M}, J)$ on which live the composite fields (2.2). Note $\mathcal{J}$ the associated gauge group.

The action of $\mathcal{J}$ on the original gauge variables is known, so its action on the composite fields obviously depends on how it affects the dressing field. The first nice possibility is as follows.

Proposition 2. Suppose the $\mathcal{K}$-dressing field u has $J$-equivariance given by $R_{j}^{*} u=j^{-1} u j$, $\forall j \in J$, or equivalently has $\mathcal{J}$-gauge transformation $u^{\gamma^{\prime}}=\gamma^{\prime-1} u \gamma^{\prime}, \forall \gamma^{\prime} \in \mathcal{J}$. Then $\omega^{u}$ is a J-principal connection on $\mathcal{P}^{\prime}$ with curvature $\Omega^{u}$, and $\varphi^{u}$ is a $J$-equivariant map on $\mathcal{P}^{\prime}$ acted upon by the covariant derivative $D^{u}=d+\rho_{*}\left(\omega^{u}\right)$. The $\mathcal{J}$-gauge transformation of the $\mathcal{K}$-invariant composite fields is then as in $(2.1)$,

$$
\begin{aligned}
& \left(\omega^{u}\right)^{\gamma^{\prime}}=\gamma^{\prime-1} \omega^{u} \gamma^{\prime}+\gamma^{\prime-1} d \gamma^{\prime}, \quad\left(\Omega^{u}\right)^{\gamma^{\prime}}=\gamma^{\prime-1} \Omega^{u} \gamma^{\prime}, \\
& \left(\varphi^{u}\right)^{\gamma^{\prime}}=\rho\left(\gamma^{\prime-1}\right) \varphi^{u} \quad \text { and } \quad\left(D^{u} \varphi^{u}\right)^{\gamma^{\prime}}=\rho\left(\gamma^{\prime-1}\right) D^{u} \varphi^{u} \text {. }
\end{aligned}
$$

The composite fields are standard gauge fields, albeit for a smaller gauge symmetry group, and are amenable to all manner of usual theoretic tortures.

The next intriguing possibility is a variation on this. Consider a map $C: \mathcal{P}^{\prime} \times J \rightarrow G$, $(p, j) \mapsto C_{p}(j)$, satisfying $C_{p}\left(j j^{\prime}\right)=C_{p}(j) C_{p j}\left(j^{\prime}\right)$. It induces a map $C\left(\gamma^{\prime}\right): \mathcal{P}^{\prime} \rightarrow G$, $p \mapsto C_{p}\left(\gamma^{\prime}(p)\right)$.

Proposition 3. Suppose the $\mathcal{K}$-dressing field $u$ has $J$-equivariance given by $R_{j}^{*} u=$ $j^{-1} u C(j), \forall j \in J$, or equivalently has $\mathcal{J}$-gauge transformation $u^{\gamma^{\prime}}=\gamma^{\prime-1} u C\left(\gamma^{\prime}\right), \forall \gamma^{\prime} \in \mathcal{J}$.

\footnotetext{
${ }^{2}$ Here 'local' is meant in the sense of field theory, not simply to express the fact that it is defined on $\mathcal{U} \in \mathcal{M}$. We refer to [13] regarding the importance of this precision.
} 
Then $\omega^{u}$ is a generalized connection (call it a twisted connection) whose curvature $\Omega^{u}$ is $A d_{C(J)}$-tensorial, and $\varphi^{u}$ is a $\rho(C(J))$-equivariant map on $\mathcal{P}^{\prime}$ acted upon by the covariant derivative $D^{u}=d+\rho_{*}\left(\omega^{u}\right)$. The $\mathcal{J}$-gauge transformation of the composite fields are then,

$$
\begin{aligned}
& \left(\omega^{u}\right)^{\gamma^{\prime}}=C\left(\gamma^{\prime}\right)^{-1} \omega^{u} C\left(\gamma^{\prime}\right)+C\left(\gamma^{\prime}\right)^{-1} d C\left(\gamma^{\prime}\right), \quad\left(\Omega^{u}\right)^{\gamma^{\prime}}=C\left(\gamma^{\prime}\right)^{-1} \Omega C\left(\gamma^{\prime}\right), \\
& \left(\varphi^{u}\right)^{\gamma^{\prime}}=\rho\left(C\left(\gamma^{\prime}\right)^{-1}\right) \varphi^{u} \quad \text { and } \quad\left(D^{u} \varphi^{u}\right)^{\gamma^{\prime}}=\rho\left(C\left(\gamma^{\prime}\right)^{-1}\right) D^{u} \varphi^{u} .
\end{aligned}
$$

The composite fields behaves as gauge fields of a new kind, implementing the gauge principle of field theory.

In both situations described, the philosophy of the DFM still applies, meaning we might want and be able to further reduce the residual gauge symmetry at hand.

Proposition 4. Suppose indeed that a $\mathcal{J}$-dressing field is available: $u^{\prime}: \mathcal{P}^{\prime} \rightarrow J$, satisfying $R_{j}^{*} u^{\prime}=j^{-1} u^{\prime}, \forall j \in J$, or $u^{\prime \gamma^{\prime}}=\gamma^{\prime-1} u^{\prime}, \forall \gamma^{\prime} \in \mathcal{J}$. It can be used to dress the composite fields (2.2) and compensate for the transformation (2.3). In order not to spoil the $\mathcal{K}$-invariance obtained after the first dressing, $u^{\prime}$ must satisfy the compatibility condition $R_{k}^{*} u^{\prime}=u^{\prime}, \forall k \in K$, or equivalently $u^{\prime \gamma}=u^{\prime}, \forall \gamma \in \mathcal{K}$.

Given such $u^{\prime}$, the map $C\left(u^{\prime}\right): \mathcal{P}^{\prime} \rightarrow G$ is a $C(J)$-dressing field satisfying $R_{j}^{*} C\left(u^{\prime}\right)=$ $C(j)^{-1} C\left(u^{\prime}\right)$ or equivalently $C\left(u^{\prime}\right)^{\gamma^{\prime}}=C\left(\gamma^{\prime}\right)^{-1} C\left(u^{\prime}\right)$. It can be used to dress the composite fields (2.2) and compensate for the transformation (2.4). In order not to spoil the $\mathcal{K}$ invariance obtained after the first dressing, the map $C(j): \mathcal{P}^{\prime} \rightarrow G$ must satisfy $R_{k}^{*} C(j)=$ $C(j)$, or equivalently $C(j)^{\gamma}=C(j)$.

In both situations, the composite fields $\chi^{u u^{\prime}}=\left(\chi^{u}\right)^{u^{\prime}}$ or $\chi^{u C\left(u^{\prime}\right)}=\left(\chi^{u}\right)^{C\left(u^{\prime}\right)}$, with $\chi=\{\omega, \Omega, \varphi\}$, are $\mathcal{H}$-invariant.

\section{Tractors and twistors from gauge reduction of the conformal Cartan geometry}

It can be shown that the conformal tractor and twistor bundles can be obtained as endproducts of gauge reduction via dressing of associated bundles of the conformal Cartan geometry, and that the standard tractor and twistor connections are really just representations of the dressed (normal) Cartan connection. ${ }^{3}$ As a matter of fact the dressing field approach has two advantages: first, it allows to generalize seamlessly tractor and twistor calculi to conformal manifolds with torsion. Secondly, it reveals that tractors and twistors are not sections of usual associated bundles coming from a representation of the structure group of a principal bundle, but that they are representatives of gauge fields of a

\footnotetext{
${ }^{3}$ Let us notice that the terms tractors and twistors nowadays assume a much broader conceptual meaning in the theory of parabolic geometries, of which conformal Cartan geometry is but one instance. According to [9] for example, any associated bundle to the principal bundle of a Cartan geometry is termed a tractor bundle. Likewise twistor theory has been generalized beyond conformal geometry. But throughout this paper we are interested in the standard conformal tractor bundle and its tractor connection as defined and constructed in [6] or [15], and in the original notion of local twistor bundle and its twistor connection essentially as presented in [16] and [17].
} 
non-standard kind as they are sections of twisted bundles constructed not through a representation but through a twisting map $C$ as described above. In the following we describe these results as synthetically as possible, and refer back to $[14,18]$ for further details.

We work over a 4-dim (spin) manifold $\mathcal{M}$. The conformal Cartan geometry is the bundle $\mathcal{P}(\mathcal{M}, H)$ whose structure group is a subgroup of the conformal group $\mathrm{SO}(2,4)-$ which preserves the metric $\Sigma=\left(\begin{array}{ccc}0 & 0 & -1 \\ 0 & \eta & 0 \\ -1 & 0 & 0\end{array}\right)$, where $\eta$ is the Minkowski metric with signature $(+---)$. It is given by $[8]$

$$
H=K_{0} \ltimes K_{1}=\left\{\left(\begin{array}{ccc}
z & 0 & 0 \\
0 & S & 0 \\
0 & 0 & z^{-1}
\end{array}\right)\left(\begin{array}{ccc}
1 & r & 1 / 2 r r^{t} \\
0 & \mathbb{1}_{4} & r^{t} \\
0 & 0 & 1
\end{array}\right) \mid z \in \mathbb{R}_{*}^{+}, S \in \mathrm{SO}(1,3), r \in \mathbb{R}^{4 *}\right\} .
$$

One has $r^{t}=\left(r \eta^{-1}\right)^{T}$ (the operation ${ }^{T}$ is the usual matrix transposition). The group $K_{1}$ is the abelian group of conformal boost, $\mathbb{R}^{4 *}$ is the dual of $\mathbb{R}^{4}$. This bundle is endowed with a Cartan connection, a $\mathfrak{g}$-valued 1-form, where $\mathfrak{g}=\mathfrak{g}_{-1}+\mathfrak{g}_{0}+\mathfrak{g}_{1}=\mathfrak{s o}(2,4),\left[\mathfrak{g}_{i}, \mathfrak{g}_{j}\right] \subset \mathfrak{g}_{i+j}$, is the graded Lie algebra of the conformal group. In matrix representation,

$$
\mathfrak{g}=\left\{\left(\begin{array}{ccc}
\varepsilon & \iota & 0 \\
\tau & s & \iota^{t} \\
0 & \tau^{t} & -\varepsilon
\end{array}\right) \mid\{\varepsilon \in \mathbb{R}, s \in \mathfrak{s o}(1,3)\} \simeq \mathfrak{g}_{0}, \quad \iota \in \mathbb{R}^{4 *} \simeq \mathfrak{g}_{1}, \quad \tau \in \mathbb{R}^{4} \simeq \mathfrak{g}_{-1}\right\} \supset \mathfrak{h}=\left\{\left(\begin{array}{ccc}
\varepsilon & \iota & 0 \\
0 & s & \iota^{t} \\
0 & 0 & -\varepsilon
\end{array}\right)\right\} .
$$

Working locally, the Cartan connection $\varpi \in \Lambda^{1}(\mathcal{U}, \mathfrak{g})$ and its curvature $\Omega$ have the matrix form $\varpi=\left(\begin{array}{ccc}a & P & 0 \\ \theta & A & P^{t} \\ 0 & \theta^{t} & -a\end{array}\right)$, where $\theta$ is the soldering form, and $\Omega=d \varpi+\varpi^{2}=\left(\begin{array}{ccc}f & C & 0 \\ \Theta & W & C^{t} \\ 0 & \Theta^{t} & -f\end{array}\right)$. The standard representation of $H$ allows to built the real vector bundle $E=\mathcal{P} \times_{H} \mathbb{R}^{6}$ whose sections are (equivalent to) $H$-equivariant maps on $\mathcal{P}$ with local expression $\varphi: \mathcal{U} \subset \mathcal{M} \rightarrow$ $\mathbb{R}^{6}$, given explicitly as column vectors $\varphi=\left(\begin{array}{c}\rho \\ \ell \\ \sigma\end{array}\right)$, where $\ell=\ell^{a} \in \mathbb{R}^{4}, \rho \in \mathbb{R}$ and $\sigma \in \mathbb{R}_{*}$. The covariant derivative on such maps is $D \varphi=d \varphi+\varpi \varphi$.

There is a complex representation of $H$, from the double cover group morphism $\bar{H} \stackrel{2: 1}{\longrightarrow}$ $H$, with

$$
\bar{H}=\bar{K}_{0} \ltimes \bar{K}_{1}:=\left\{\left(\begin{array}{cc}
z^{1 / 2} \bar{S}^{-1 *} & 0 \\
0 & z^{-1 / 2} \bar{S}
\end{array}\right)\left(\begin{array}{cc}
\mathbb{1}_{2} & -i \bar{r} \\
0 & \mathbb{1}_{2}
\end{array}\right) \mid z \in \mathbb{R}_{*}^{+}, \bar{S} \in \mathrm{SL}(2, \mathbb{C}), \bar{r} \in \operatorname{Herm}(2, \mathbb{C})\right\} .
$$

It is induced by the vector space isomorphism $\mathbb{R}^{4} \rightarrow \operatorname{Herm}(2, \mathbb{C})=\left\{M \in M_{2}(\mathbb{C}) \mid M^{*}=\right.$ $M\}, x \mapsto \bar{x}=x^{a} \sigma_{a}$ - where ${ }^{*}$ means transconjugation, $\sigma_{0}=\mathbb{1}_{2}$ and $\sigma_{k=\{1,2,3\}}$ are Pauli matrices - and by the double covering group morphism $\operatorname{Spin}(1,3) \simeq \mathrm{SL}(2, \mathbb{C}) \stackrel{2: 1}{\longrightarrow} \mathrm{SO}(1,3)$, $\bar{S} \mapsto S$. This also induces the double cover $\mathrm{Spin}(2,4) \simeq \mathrm{SU}(2,2) \stackrel{2: 1}{\longrightarrow} \mathrm{SO}(2,4)$, where $\mathrm{SU}(2,2)$ is the group preserving the metric $\bar{\Sigma}=\left(\begin{array}{cc}0 & \mathbb{1}_{2} \\ \mathbb{1}_{2} & 0\end{array}\right)$, which in turn implies the Lie algebra isomorphism $\mathfrak{s o}(2,4)=\mathfrak{g} \rightarrow \mathfrak{s u}(2,2)=\overline{\mathfrak{g}}$ with

$$
\overline{\mathfrak{g}}=\left\{\left(\begin{array}{cc}
-\left(\bar{s}^{*}-\varepsilon / 2 \mathbb{1}\right) & -i \bar{\iota} \\
i \bar{\tau} & \bar{s}-\varepsilon / 2 \mathbb{1}
\end{array}\right) \mid \varepsilon \in \mathbb{R}, \bar{s} \in \mathfrak{s l}(2, \mathbb{C}) \text { and } \bar{\iota}, \bar{\tau} \in \operatorname{Herm}(2, \mathbb{C})\right\} .
$$

The Cartan connection then has the complex representation $\bar{\varpi}=\left(\begin{array}{cc}-\left(\bar{A}^{*}-a / 2 \mathbb{1}\right) & -i \bar{P} \\ i \bar{\theta} & \bar{A}-a / 2 \mathbb{1}\end{array}\right)$, and similarly its curvature is $\bar{\Omega}=\left(\begin{array}{cc}-\left(\bar{W}^{*}-f / 2 \mathbb{1}\right) & -i \bar{C} \\ i \bar{\Theta} & \bar{W}-f / 2 \mathbb{1}\end{array}\right)$. The associated complex vector bundle $\mathrm{E}=\mathcal{P} \times \overline{\mathbf{H}} \mathbb{C}^{4}$ has sections equivalent to $\bar{H}$-equivariant maps on $\mathcal{P}$ with local expression 
$\psi: \mathcal{U} \subset \mathcal{M} \rightarrow \mathbb{C}^{4}$, given explicitly as $\psi=\left(\begin{array}{c}\pi \\ \omega\end{array}\right)$, where $\pi$ and $\omega \in \mathbb{C}^{2}$ are dual Weyl spinors. The covariant derivative on such maps is $\bar{D} \psi=d \psi+\bar{\varpi} \psi$.

As a matter of fact, bare sections of $E$ and $E$ are not yet, respectively, tractors and twistors (keeping in mind our remark in footnote 3). This is clear when one inspects the gauge transformations of $\varphi$ and $\psi$ under $\mathcal{K}_{0}=\mathcal{S O} \times \mathcal{W} \subset \mathcal{H}$ - where $\mathcal{W}=\left\{Z: \mathcal{U} \rightarrow W \subset K_{0} \mid Z=\left(\begin{array}{ccc}z & 0 & 0 \\ 0 & \mathbb{1}_{4} & 0 \\ 0 & 0 & z^{-1}\end{array}\right)\right\}$ is the group of Weyl rescalings - and compare with the transformation laws of tractors and twistors in the literature: they are nothing alike. Curiously, the correct laws can be recovered after a gauge symmetry reduction via dressing. ${ }^{4}$

Indeed, from the Cartan connection itself one builds a (local) $\mathcal{K}_{1}$-dressing field $u_{1}$ : $\mathcal{U} \rightarrow K_{1}$, explicitly $u_{1}=\left(\begin{array}{ccc}1 & q & 1 / 2 q q^{t} \\ 0 & \mathbb{1} & q^{t} \\ 0 & 0 & 1\end{array}\right)$ with $q=q_{a}=a_{\mu} e^{\mu}{ }_{a}$, where $a=a_{\mu} d x^{\mu}$ and $\theta^{a}=$ $e^{a}{ }_{\mu} d x^{\mu}$ is the soldering form whose components are the tetrad field. From the $\mathcal{K}_{1}$-gauge transformation of $\varpi$ one checks that $u_{1}^{\gamma_{1}}=\gamma_{1}^{-1} u_{1}, \forall \gamma_{1} \in \mathcal{K}_{1}$, as is defining of a dressing field. One then simply apply Proposition 1 and built the $\mathcal{K}_{1}$-invariant composite fields

$$
\begin{aligned}
\varpi_{1} & :=u_{1}^{-1} \varpi u_{1}+u_{1}{ }^{-1} d u_{1}, & & & \Omega_{1} & =d \omega_{1}+\omega_{1}^{2}, \\
\varphi_{1} & :=u_{1}^{-1} \varphi, & & \text { and } & D_{1} \varphi_{1} & =d \varphi_{1}+\varpi_{1} \varphi_{1}, \\
\psi_{1} & :=\bar{u}_{1}^{-1} \psi, & & \text { and } & \bar{D}_{1} \psi_{1} & =d \psi_{1}+\bar{\varpi}_{1} \psi_{1} .
\end{aligned}
$$

Their residual gauge symmetry group is obviously $\mathcal{K}_{0}$, but what is the explicit transformation law? The undressed field variables being standard gauge fields, we only need to find the $\mathcal{K}_{0}$ transformation of the dressing field $u_{1}$.

Given the gauge transformation of $\varpi$ under $\mathcal{S O}=\left\{\mathrm{S}: \mathcal{U} \rightarrow \mathrm{SO}(1,3) \mid \mathrm{S}=\left(\begin{array}{ccc}1 & 0 & 0 \\ 0 & S & 0 \\ 0 & 0 & 1\end{array}\right)\right\}$, one finds that $u_{1}^{S}=\mathrm{S}^{-1} \mathrm{u}_{1} \mathrm{~S}$. This is an instance of Proposition 2, so one concludes that the above composite fields are genuine standard $\mathcal{S O}$-gauge fields with transformations given by the usual relations (2.3).

Following the same logic by considering next the gauge transformation of $\varpi$ under $\mathcal{W}$, one easily finds that $u_{1}^{Z}=Z^{-1} u_{1} C(z)$ where the twisting map $C(z): \mathcal{U} \subset \mathcal{M} \rightarrow K_{1} W \subset H$ is explictly given as $C(z):=\left(\begin{array}{ccc}z & \Upsilon(z) & z^{-1} / 2 \Upsilon(z)^{2} \\ 0 & \mathbb{1}_{4} & z^{-1} \Upsilon(z)^{t} \\ 0 & 0 & z^{-1}\end{array}\right)$, with $\Upsilon(z)=\Upsilon(z)_{a}:=z^{-1} \partial_{\mu} z e^{\mu}{ }_{a}$, and $\Upsilon(z)^{2}=\Upsilon(z) \Upsilon(z)^{t}$. Notice that $C\left(z^{\prime}\right) C(z) \neq C\left(z^{\prime} z\right)$, but instead one has $C\left(z^{\prime} z\right)=$ $C\left(z^{\prime}\right) Z^{\prime-1} C(z) Z^{\prime}$. It is a particular case of Proposition 3 , so one concludes that the composite fields (3.2) are non-standard $\mathcal{W}$-gauge fields with transformations given by (2.4). Furthermore, the relation $\varphi_{1}^{Z}=C(z)^{-1} \varphi_{1}$ is precisely the right rescaling law for a tractor field, and $\psi_{1}^{Z}=\bar{C}(z)^{-1} \psi_{1}$ - with $\bar{C}(z)=\left(\begin{array}{cc}z^{1 / 2} \mathbb{1}_{2} & -i z^{-1 / 2} \bar{\Upsilon}(z) \\ 0 & z^{-1 / 2} \mathbb{1}_{2}\end{array}\right)$ - is the rescaling law for a twistor.

The immediate implication is that $\varpi_{1}$ is a generalization of the so-called tractor connection, and that $\bar{\varpi}_{1}$ is likewise a generalization of the twistor connection, since their

\footnotetext{
${ }^{4}$ Alternatively, the transformation law for the standard tractor connection (and tractors) can be recovered via the notion of Weyl structure, a concept associated to any parabolic geometries. The reader can consult chapter 5 of [9] for further details, and is invited to weigh up the respective merits of both approaches. A proof of formal equivalence between the data of a Weyl structure and of a dressing field (as described in Proposition 2) for $|1|$-graded parabolic geometries is given in appendix A below.
} 
torsion $\Theta$ is non zero. Only when one dresses the (unique) normal Cartan connection $\varpi_{N}$ — depending only on the d.o.f of $\theta$ [8] — do we end-up with the usual tractor $\varpi_{N, 1}$ and twistor $\bar{\varpi}_{N, 1}$ connections. Explicitly, $\varpi_{N, 1}=\left(\begin{array}{ccc}0 & P_{1} & 0 \\ \theta & A_{1} & P^{t} \\ 0 & \theta^{t} & 0\end{array}\right)$ with $\theta$ the soldering form, $A_{1}$ the Lorentz/spin connection, and $P_{1}$ the Schouten 1-form. The curvature is $\Omega_{N, 1}=\left(\begin{array}{ccc}0 & C_{1} & 0 \\ 0 & W_{1} & C_{1}^{t} \\ 0 & 0 & 0\end{array}\right)$, with $W_{1}$ the Weyl 2-form and $C_{1}$ the Cotton 2-form. Again, we refer to $[14,18]$ for a more detailed account.

Notice that the dressed Cartan connection induces a conformal class of metric $c=[g]$ on $\mathcal{M}$ via its soldering part. Indeed if $\varpi_{1}$ induces, via $\theta$, the metric $g:=e^{T} \eta e$, then $\varpi_{1}^{Z}$ induces via $\theta^{Z}=z \theta$ the metric $z^{2} g$. Nevertheless it is the normal Cartan geometry $\left(\mathcal{P}, \varpi_{N, 1}\right)$ which is truly equivalent to the data of the conformal manifold $(\mathcal{M}, c)$.

\section{A dressing field for the Weyl gauge symmetry}

So far so good. Interestingly, it is possible to perform still another gauge reduction on this tractor-twistor geometric set-up: this time we can erase the Weyl gauge symmetry. The explicit Weyl rescaling law for the tractor field $\varphi_{1}=\left(\begin{array}{c}\rho_{1} \\ \ell_{1}\end{array}\right)$ is: $\varphi_{1}^{Z}=C(z)^{-1} \varphi_{1}=$ $\left(\begin{array}{c}z^{-1}\left(\rho_{1}-\Upsilon(z) \ell_{1}+\sigma / 2 \Upsilon(z)^{2}\right) \\ \ell_{1}-\Upsilon(z)^{t} \sigma \\ z \sigma\end{array}\right)$. Remark that the trator field supplies a candidate dressing field for the Weyl gauge symmetry, otherwise known as a dilaton. Indeed defining $\phi:=\sigma^{-1}$, we have $\phi^{z}=z^{-1} \phi, \forall z: \mathcal{U} \rightarrow \mathbb{R}_{*}^{+}$, which is the defining property of a dressing field. It is by the way clear that $\phi^{\gamma_{1}}=\phi$. Also, $C(z)$ depends only on $e=e^{a}{ }_{\mu}$ which satisfies $e^{\gamma_{1}}=e$, so that $C(z)^{\gamma_{1}}=C(z)$. Therefore, by Proposition 3 the map $C(\phi): \mathcal{U} \rightarrow K_{1} W$, given by $C(\phi)=\left(\begin{array}{ccc}\phi & \Upsilon(\phi) & \phi^{-1} / 2 \Upsilon(\phi)^{2} \\ 0 & \mathbb{1}_{4} & \phi^{-1} \Upsilon(\phi)^{t} \\ 0 & 0 & \phi^{-1}\end{array}\right)$ with $\Upsilon(\phi)=\Upsilon(\phi)_{a}:=\phi^{-1} \partial_{\mu} \phi e^{\mu}{ }_{a}$, satisfies both $C(\phi)^{\mathcal{W}}=C(z)^{-1} C(\phi)$ and $C(\phi)^{\gamma_{1}}=C(\phi)$. It is thus a dressing field adequate for the purpose of erasing the residual Weyl gauge symmetry of the composite fields (3.2) while preserving their conformal boost invariance. Applying then again Proposition 1, eq. (2.2), one defines the following $\mathcal{K}_{1}$ - and $\mathcal{W}$-invariant composite fields

$$
\begin{aligned}
& \varpi:=C(\phi)^{-1} \varpi_{1} C(\phi)+C(\phi)^{-1} d C(\phi), \quad \boldsymbol{\Omega}=d \varpi+\varpi^{2}, \\
& \boldsymbol{\varphi}:=C(\phi)^{-1} \varphi_{1}, \quad \text { and } \quad \boldsymbol{D} \boldsymbol{\varphi}=d \boldsymbol{\varphi}+\varpi \boldsymbol{\varphi}, \\
& \boldsymbol{\psi}:=\bar{C}(\phi)^{-1} \psi_{1}, \quad \text { and } \quad \overline{\boldsymbol{D}} \boldsymbol{\psi}=d \boldsymbol{\psi}+\bar{\varpi} \boldsymbol{\psi} .
\end{aligned}
$$

We have explicitly $\varpi=\left(\begin{array}{lll}0 & \boldsymbol{P} & 0 \\ \boldsymbol{\theta} & \boldsymbol{A} & \boldsymbol{P}^{t} \\ 0 & \boldsymbol{\theta}^{t} & 0\end{array}\right)=\left(\begin{array}{ccc}0 & \phi^{-1}\left(P_{1}+\nabla \Upsilon(\phi)-\Upsilon(\phi) \theta \Upsilon(\phi)+1 / 2 \Upsilon(\phi)^{2} \theta^{t}\right) & 0 \\ \phi \theta & A_{1}+\theta \Upsilon(\phi)-\Upsilon(\phi)^{t} \theta^{t} & * \\ 0 & \phi \theta^{t} & 0\end{array}\right)$, which induces via its Weyl-invariant soldering form $\boldsymbol{\theta}$ the invariant Lorentzian metric $\boldsymbol{g}=\phi^{2} g$ on $\mathcal{M}$. Also, the invariant tractor field is now $\varphi=\left(\begin{array}{c}\rho \\ \ell \\ 1\end{array}\right)$, and has manifestly one less d.o.f than its undressed counterpart.

Quite obviously, there now remains only the Lorentz gauge group $\mathcal{S O}$. The question is how the fields (4.1) behaves w.r.t. this residual symmetry. Since it is already established 
that the $\mathcal{K}_{1}$-invariant fields (3.2) are genuine standard $\mathcal{S O}$-gauge fields, it is only necessary to consider the Lorentz transformation of the dressing field $C(\phi)$. It is easily checked that $C(\phi)^{\mathrm{S}}=\mathrm{S}^{-1} C(\phi) \mathrm{S}$, so by Proposition 2 the $\mathcal{K}_{1}$ - and $\mathcal{W}$-invariant composite fields (4.1) are genuine standard $\mathcal{S O}$-gauge fields, therefore transforming according to (2.3):

$$
\begin{array}{rlrlrl}
\varpi^{\mathrm{S}} & =\mathrm{S}^{-1} \varpi \mathrm{S}+\mathrm{S}^{-1} d \mathrm{~S}, & & \boldsymbol{\Omega}^{\mathrm{S}} & =\mathrm{S}^{-1} \boldsymbol{\Omega S}, \\
\boldsymbol{\varphi}^{\mathrm{S}} & =\mathrm{S}^{-1} \boldsymbol{\varphi}, & \text { and } & & (\boldsymbol{D} \boldsymbol{\varphi})^{\mathrm{S}} & =\mathrm{S}^{-1} \boldsymbol{D} \boldsymbol{\varphi}, \\
\boldsymbol{\psi}^{\mathrm{S}} & =\overline{\mathrm{S}}^{-1} \boldsymbol{\psi}, & \text { and } & (\overline{\boldsymbol{D}} \boldsymbol{\psi})^{\mathrm{S}} & =\overline{\mathrm{S}}^{-1} \overline{\boldsymbol{D}} \boldsymbol{\psi} .
\end{array}
$$

At this point, several interesting observations can be made. First, it is striking that the Weyl-dressed twistor $\boldsymbol{\psi}$, which now only supports Lorentz gauge transformations via $\overline{\mathcal{S O}}=\left\{\overline{\mathrm{S}}: \mathcal{U} \rightarrow \mathrm{SL}(2, \mathbb{C}) \oplus \mathrm{SL}(2, \mathbb{C})^{*} \mid \bar{S}=\left(\begin{array}{cc}\bar{S}^{-1 *} & 0 \\ 0 & \bar{S}\end{array}\right)\right\}$, can then be interpreted as a Dirac spinor field.

Furthermore, let us highlight - even if that might seem obvious to some readers - that the underlying geometric set-up of the twistor sector provides a preferred choice of basis for gamma matrices. Indeed, the Lie algebra isomorphism $\mathfrak{s o}(2,4) \rightarrow \mathfrak{s u}(2,2)$ necessary for the construction of the twistor sector induces the vector space isomorphism $\mathbb{R}^{4} \rightarrow$ $\overline{\mathfrak{g}}_{-1} \oplus \overline{\mathfrak{g}}_{1} \subset \mathfrak{s u}(2,2)$, given explicitly by $x=x^{a} b_{a} \mapsto\left(\begin{array}{cc}0 & i \bar{x}^{t} \\ i \bar{x} & 0\end{array}\right)=\frac{i}{\sqrt{2}} x^{a}\left(\begin{array}{cc}0 & \tilde{\sigma}_{a} \\ \sigma_{a} & 0\end{array}\right)=\frac{i}{\sqrt{2}} x^{a} \gamma_{a}$. The gamma matrices $\gamma_{a}:=\left\{\gamma_{0}=\left(\begin{array}{cc}0 & \sigma_{0} \\ \sigma_{0} & 0\end{array}\right), \gamma_{k}=\left(\begin{array}{cc}0 & -\sigma_{k} \\ \sigma_{k} & 0\end{array}\right)_{\mid k=1,2,3}\right\}$ are otherwise known as the Weyl — or chiral - basis. From this isomorphism follows that they Lorentz transform via $\gamma_{a}^{\mathrm{S}}=\left(S^{-1}\right)^{b}{ }_{a} \gamma_{b}=\overline{\mathrm{S}}^{-1} \gamma_{a} \overline{\mathrm{S}}$. As it should, they satisfy the Clifford algebra relation $\left\{\gamma_{a}, \gamma_{b}\right\}=2 \eta_{a b} \mathbb{1}_{4}$. The hermiticity properties $\gamma_{0}^{*}=\gamma_{0}$ and $\gamma_{k}^{*}=-\gamma_{k}$, are here necessary and do not result from an arbitrary convenient choice (as is sometimes the case in the physics literature). The fact that $\frac{1}{2}\left[\gamma_{a} \gamma_{b}\right]$ - the so-called spin operator $\sigma_{a b}[19]$ - is a basis of $\mathfrak{s l}(2,) \oplus \mathfrak{s l}(2, \mathbb{C})^{*} \subset \overline{\mathfrak{g}}_{0}$ simply reflects the graded structure of $\mathfrak{s u}(2,2)=\overline{\mathfrak{g}}$ : $\left[\overline{\mathfrak{g}}_{-1}, \overline{\mathfrak{g}}_{1}\right] \subset \overline{\mathfrak{g}}_{0}$. The fifth gamma matrix $\gamma_{5}:=i \gamma_{0} \gamma_{1} \gamma_{2} \gamma_{3}=\left(\begin{array}{cc}\mathbb{1}_{2} & 0 \\ 0 & -\mathbb{1}_{2}\end{array}\right)$ satisfies $\gamma_{5}^{*}=\gamma_{5}$. By the way, $\gamma^{a}:=\eta^{a b} \gamma_{b}=\left(\begin{array}{cc}0 & \sigma_{a} \\ \tilde{\sigma}_{a} & 0\end{array}\right)=\left\{\gamma^{0}=\gamma_{0}=\left(\begin{array}{cc}0 & \sigma_{0} \\ \sigma_{0} & 0\end{array}\right), \gamma^{k}=-\gamma_{k}=\left(\begin{array}{cc}0 & \sigma_{k} \\ -\sigma_{k} & 0\end{array}\right)_{\mid k=1,2,3}\right\}$, so $\gamma^{5}=-\gamma_{5}$. As is then usual, one defines the chiral projectors $P_{L}:=\frac{1}{2}\left(\mathbb{1}_{4}+\gamma_{5}\right)$ and $P_{R}:=\frac{1}{2}\left(\mathbb{1}_{4}-\gamma_{5}\right)$, so that $\boldsymbol{\psi}_{L}=P_{L} \boldsymbol{\psi}=\left(\begin{array}{c}\boldsymbol{\pi} \\ 0\end{array}\right)$ and $\boldsymbol{\psi}_{R}=P_{R} \boldsymbol{\psi}=\left(\begin{array}{c}0 \\ \boldsymbol{\omega}\end{array}\right)$. By a slight abuse of notation one can then rewrites the Weyl-invariant twistor like a Dirac spinor $\boldsymbol{\psi}=\left(\begin{array}{l}\boldsymbol{\psi}_{L} \\ \boldsymbol{\psi}_{R}\end{array}\right)$.

Notice that the Lorentz part of the dressed Cartan connection $\varpi, \mathbf{A}=\left(\begin{array}{lll}0 & 0 & 0 \\ 0 & A & 0 \\ 0 & 0 & 0\end{array}\right)$, maps to the complex representation $\overline{\mathbf{A}}=\left(\begin{array}{cc}-\overline{\boldsymbol{A}}^{*} & 0 \\ 0 & \overline{\boldsymbol{A}}\end{array}\right)$. We have therefore the Lorentz covariant derivative acting on spinors: $\mathbf{D} \boldsymbol{\psi}:=d \boldsymbol{\psi}+\overline{\mathbf{A}} \boldsymbol{\psi}$, as a piece of the invariant Cartan derivative $\overline{\boldsymbol{D}} \boldsymbol{\psi}$. Defining the gamma 1-form $\boldsymbol{\gamma}=\gamma_{a} \boldsymbol{\theta}^{a}=\gamma_{a} \boldsymbol{e}_{\mu}^{a} d x^{\mu}=: \gamma_{\mu} d x^{\mu}$ - where $\gamma_{\mu}$ are sometimes called "curved-space gamma matrices" and satisfy $\left\{\gamma_{\mu}, \gamma_{\nu}\right\}=2 \boldsymbol{g}_{\mu \nu} \mathbb{1}_{4}$ - one can build the Dirac operator $m$-form $\not \boldsymbol{\psi}=\gamma \wedge{ }_{g} \mathbf{D} \boldsymbol{\psi}=\gamma^{\mu} \mathbf{D}_{\mu} \boldsymbol{\psi}$ vol, where $* \boldsymbol{g}$ is the Hodge star operator associated with the invariant Lorentzian metric $\boldsymbol{g}$ and vol $=\sqrt{|\boldsymbol{g}|} d x^{4}$ is the volume form on $\mathcal{M}$. Thanks to the group metric $\bar{\Sigma}$ of $\mathrm{SU}(2,2)$, one can then naturally write a Lagrangian for the (massless) Dirac field $\boldsymbol{\psi}: L_{\text {Dirac }}(\boldsymbol{\psi}, \overline{\mathbf{A}})=\langle\boldsymbol{\psi}, \boldsymbol{D} \psi\rangle=\boldsymbol{\psi}^{*} \bar{\Sigma} \boldsymbol{\emptyset} \boldsymbol{\psi}=\overline{\boldsymbol{\psi}} \boldsymbol{\emptyset} \boldsymbol{\psi}$. Notice that the usual definition for the Dirac adjoint spinor $\overline{\boldsymbol{\psi}}=\boldsymbol{\psi}^{*} \boldsymbol{\gamma}^{0}$ — initially suggested 
by Bargmann instead of the problematic curved-space version $\boldsymbol{\psi}^{*} \boldsymbol{\gamma}^{a}\left(\boldsymbol{e}^{-1}\right)_{a}^{0}[20]^{5}$ - is automatic here due to the welcomed fact that $\gamma^{0}=\bar{\Sigma}{ }^{6}$

Let us pause to take stock of our main results. We considered the most general geometric framework for conformal gauge theory, i.e. the conformal Cartan bundle $\mathcal{P}(\mathcal{M}, H)$ endowed with a Cartan connection $\varpi$ and the naturally associated real $E$ and complex $\mathrm{E}$ vector bundles. We showed that a first symmetry reduction via dressing allows to recover the standard conformal tractor bundle $E^{u_{1}}=\mathcal{T}$ and twistor bundles $\mathrm{E}^{u_{1}}=\mathrm{T}$ where tractor and twistor connections (with torsion) are naturally induced by the dressed Cartan connection $\varpi_{1}$. Then we observed that the tractor field $\varphi_{1}$ provides a dilaton, which is a local Weyl-dressing field, so that a Weyl gauge symmetry reduction via dressing is possible. As output we obtain $\boldsymbol{E}$ whose sections are Weyl-invariant tractors, and $\mathbf{E}$ whose sections are Weyl-invariant twistors, which actually have all the characteristics of Dirac spinors describing fermion fields. We are left with only a residual Lorentz gauge symmetry as relevant, and no SSB was necessary: the symmetry reductions were non-dynamically achieved, via dressing. The import of this fact is discussed in section 5. But before this, let us offer a simple illustrative toy model for the reader's consideration.

It is a striking fact that the $\mathcal{S U}(2) \times \mathcal{U}(1)$ electroweak model can be treated via the DFM: a dressing field is extracted from the $\mathbb{C}^{2}$-scalar field, allowing to erase $\mathcal{S U}(2)$ and leaving only a $\mathcal{U}(1)$ residual gauge symmetry. The (dressed) scalar field embedded in a potential gives mass to the (dressed) gauge and matter fields via its (unique) non-vanishing VEV. The notion of SSB never appears here. See $[12,21]$ for a technical account and [13] for a philosophical discussion of this alternative approach. Given the analogy with the present situation, one can consider the most natural Lagrangian for the conformal Cartan geometry that would imitate the electroweak model:

$$
L(\varpi, \varphi, \psi)=\frac{1}{2} \operatorname{Tr}\left(\Omega \wedge *_{\boldsymbol{g}} \Omega\right)+\left\langle D \varphi, *_{\boldsymbol{g}} D \varphi\right\rangle-V(\varphi)+\left\langle\psi,{ }_{\boldsymbol{g}} \bar{D} \phi \psi\right\rangle-|\varphi|\left\langle\psi,{ }_{\boldsymbol{g}} \psi\right\rangle .
$$

The $\mathbb{R}^{6}$-field $\varphi$ is the analogue to the $\mathbb{C}^{2}$-scalar field and is embedded in the potential $V(\varphi)=\left(\alpha\langle\varphi, \varphi\rangle+\beta\langle\varphi, \varphi\rangle^{2}\right)$ vol, where for the usual reasons $\alpha$ is an unconstrained real parameter while $\beta>0$. The last term is a Yukawa-type coupling where $|\varphi|^{2}:=\langle\varphi, \varphi\rangle=$ $\varphi^{T} \Sigma \varphi$

A priori this Lagrangian describes a conformal $\mathcal{H}=\mathcal{K}_{0} \ltimes \mathcal{K}_{1}$-gauge theory. But according to our discussion following Proposition 1, given that there is a $\mathcal{K}_{1}$-dressing field $u_{1}$, the Lagrangian can be rewritten as

$$
L\left(\varpi_{1}, \varphi_{1}, \psi_{1}\right)=\frac{1}{2} \operatorname{Tr}\left(\Omega_{1} \wedge *_{\boldsymbol{g}} \Omega_{1}\right)+\left\langle D_{1} \varphi_{1}, *_{\boldsymbol{g}} D_{1} \varphi_{1}\right\rangle-V\left(\varphi_{1}\right)+\left\langle\psi_{1}, *_{\boldsymbol{g}} \bar{D}_{1} \psi_{1}\right\rangle-\left|\varphi_{1}\right|\left\langle\psi_{1}, *_{\boldsymbol{g}} \psi_{1}\right\rangle,
$$

which is a $\mathcal{K}_{0}$-gauge theory involving the tractor field $\varphi_{1}$ and the twistor field $\psi_{1}$, both a priori massless.

\footnotetext{
${ }^{5}$ As quoted by Pauli in [33] p.340 (in German). A textbook account is [34] p.268. See also [19] or [35].

${ }^{6}$ An incidental fact that should not obscure the conceptual distinction that $\gamma_{a}$ are linear operators on the space of spinors while $\bar{\Sigma}$ defines an hermitian form on it. This speaks in favor of the view proposed here.
} 
But it is not over yet. Given that we also have a dressing field for the Weyl symmetry, the 'super-dilaton' $C(\phi)$ (with value in $K_{1} W \subset H$ ), the Lagrangian is further rewritten as

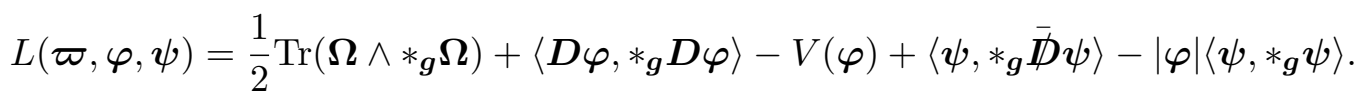

It is actually a $\mathcal{S O}$-gauge theory! It involves a Weyl-invariant tractor $\boldsymbol{\varphi}$ and the Dirac spinor $\boldsymbol{\psi}$, both coupled to a Weyl-invariant conformal-type gravitational field $\varpi$ whose dynamics is given by the Yang-Mills-type term. ${ }^{7}$ The potential function $V: \mathbb{R}^{6} \rightarrow \mathbb{R}$ has differential (in the sense of multivariable calculus) $d V_{\varphi}=2(\alpha+2 \beta\langle\varphi, \varphi\rangle)\langle\varphi|$. The potential is thus extremal either for $\varphi=0$ or for $\varphi_{0}$ s.t $\left\langle\boldsymbol{\varphi}_{0}, \boldsymbol{\varphi}_{0}\right\rangle=-\frac{\alpha}{2 \beta}$. The minimum of the potential is reached for the latter, which is then the so-called vacuum expectation value (VEV). The invariant tractor $\varphi$ therefore endows both the gravitational and matter fields with masses through its non vanishing VEV. In particular the Dirac spinor $\boldsymbol{\psi}$ has a mass $m=\sqrt{-\frac{\alpha}{2 \beta}}$.

Notice that this is achieved not by breaking the Weyl symmetry, which is erased via dressing, but via spontaneous breaking of the only physically relevant symmetry in the model: the Lorentz gauge symmetry. A phenomenon for which there is also room for in some models describing the Planck era of the early Universe in string theory, loop quantum cosmology, and even the more conservative Standard Model Extension (SME), and whose low energy relics could be within experimental reach [22-24]. Since Lorentz violation is related to CPT violation [25], it could be part of the explanation of the matter-antimatter asymmetry in the Big Bang baryogenesis $[26,27] .{ }^{8}$

\section{Discussion}

The above Lagrangian is quite natural but obviously a rough toy model, proposed mostly to sharpen the skepticism of the reader interested in Weyl/scale invariant models where the Weyl gauge symmetry is subsequently spontaneously broken. This toy model shows that one can have a Weyl symmetry that is neither spontaneously broken nor gauge fixed, but erased by an adequate redistribution of the d.o.f via dressing.

In this respect, and as a comment on existing literature, we highlight that our main results support the analysis of Jackiw and $\mathrm{Pi}$ [11] who assert that the Weyl symmetry in recently introduced cosmological models, notably [2], is "fake" and has no dynamical consequences. They supported their claim by showing that in these models the Noether current associated with the Weyl symmetry vanishes. But this same argument would lead to claim that the Weyl symmetry in Weyl/conformal gravity is fake as well: indeed, almost simultaneously Campigotto and Fatibene have shown [28] that the Noether current also

\footnotetext{
${ }^{7}$ The latter reduces to the standard Weyl-squared gravity $\frac{1}{2} \operatorname{Tr}\left(\boldsymbol{W} \wedge *_{g} \boldsymbol{W}\right)$ when we restrict to the normal Cartan connection.

${ }^{8}$ In this respect, one remarks that another natural term that could be added in the Lagrangian (4.5) (but not in (4.3) and (4.4) since it would explicitly break the $\mathcal{K}_{1}$ and $\mathcal{W}$ symmetries) is $-\langle\boldsymbol{\psi}, \bar{\varphi} \boldsymbol{\psi}\rangle$, where the invariant tractor $\varphi$ is mapped to $\bar{\varphi}:=\left(\begin{array}{cc}\rho \mathbb{1}_{2} & i \overline{\ell^{t}} \\ i \bar{\ell} & \mathbb{1}_{2}\end{array}\right)$ so that $\mathrm{S}^{-1} \varphi \mapsto \overline{\mathrm{S}}^{-1} \bar{\varphi} \overline{\mathrm{S}}$. It contains the term $\frac{-i}{\sqrt{2}} \ell^{a}\left\langle\boldsymbol{\psi}, \boldsymbol{\gamma}_{a} \boldsymbol{\psi}\right\rangle=\frac{-i}{\sqrt{2}} \ell^{a} \overline{\boldsymbol{\psi}} \boldsymbol{\gamma}_{a} \boldsymbol{\psi}$ which is the kind of Lorentz and CPT breaking terms appearing in the SME.
} 
vanishes there. In our view Jackiw and $\mathrm{Pi}$ are right, but the crucial argument is that in the models they discuss, a local dressing field - the dilaton - could be used to erase the Weyl symmetry, while there is no such dressing field in Weyl gravity. As they themselves remark at the end of their paper:

Introducing a spurion field and dressing up a model to appear gauge invariant is what we call a fake gauge invariance. [...] It will be interesting to find the symmetry current in a conventional Weyl invariant model, built on the square of the Weyl tensor. There the symmetry is again local, but no scalar field is present to absorb the "gauge freedom."

What they call a fake gauge symmetry is otherwise known as an artificial gauge symmetry in philosophy of physics. In this field, the distinction artificial vs substantial gauge symmetries is very significant and a direct outgrowth of the long standing reflexion regarding the physical meaning of general covariance in General Relativity that was required to address the notorious Kretschmann objection $[29,30]$. It happens that the DFM provides a simple criterion to detect artificial gauge symmetries: If a theory contains a local dressing field, then its gauge symmetry is artificial [13]. In that regard, Jackiw and Pi correctly point out that in the models under study, the dilaton is a local dressing field (which they call a spurion field) so that the Weyl symmetry is artificial, or fake. Whereas in Weyl/conformal gravity there is no such local dressing field, so its Weyl symmetry is a substantial gauge symmetry. Similar observations in the same period have been made by Hertzberg [31] and are likewise plainly interpreted within the framework of the DFM and the present work. More recent papers by 't Hooft [3, 32], where Weyl symmetry is supposed to be spontaneously broken, are also vulnerable to the same objection: The dilaton introduced is a local dressing field, the Weyl symmetry of the model is then artificial and is therefore of questionable physical meaning. We hope to have provided and illustrated here a handy diagnostic tool to detect spurious gauge symmetries that might occur in model building, be it in cosmology or beyond SM physics.

\section{Acknowledgments}

The author thanks Roberto Percacci (SISSA, Italy) for drawing his attention to the paper [11] by Jackiw and Pi. Credit is due to a careful anonymous reviewer whose remarks and suggestions helped improve the paper. This work was supported by a postdoctoral grant from the Complexys Institut of the University of Mons, Belgium.

\section{A Dressing fields and Weyl structures}

Here, we show that for a $|1|$-graded parabolic Cartan geometry, the notions of Weyl structure and of dressing field with residual equivariance as specified in Proposition 2 are formally equivalent.

Let us first describe a $|1|$-graded parabolic geometry. Suppose we have a group $G$ with graded Lie algebra $\mathfrak{g}=\mathfrak{g}_{-1}+\mathfrak{g}_{0}+\mathfrak{g}_{1}$, let $H \subset G$ with Lie $H=\mathfrak{g}_{0}+\mathfrak{g}_{1}$ be the parabolic 
subgroup. The principal bundle $\mathcal{P}(\mathcal{M}, H)$ is a parabolic geometry of type $(G, H)$. The conformal Cartan geometry is an example of $|1|$-graded parabolic geometry.

Now, the subbundle $\mathcal{P}_{0}\left(\mathcal{M}, G_{0}\right)$, where $\operatorname{Lie} G_{0}=\mathfrak{g}_{0}$, is realized as $\mathcal{P}_{0}=\mathcal{P} / K$ where Lie $K=\mathfrak{g}_{1}$ and $K \triangleleft H$. So there is a principal bundle $\mathcal{P} \rightarrow \mathcal{P}_{0}$ with structure group $K$. A (global) Weyl structure for the parabolic geometry $\mathcal{P}$ is a global section $\sigma: \mathcal{P}_{0} \rightarrow \mathcal{P}$ with $G_{0}$-equivariance $R_{g}^{*} \sigma=R_{g} \circ \sigma, \forall g \in G_{0}$. Such a global Weyl structure for a parabolic geometry always exists (see section 5.1 in [9]).

A $K$-dressing field with residual equivariance as in Proposition 2 is a map $u: \mathcal{P} \rightarrow K$ with $K$-equivariance $R_{k}^{*} u=k^{-1} u, \forall k \in K$, and with $G_{0}$-equivariance $R_{g}^{*} u=g^{-1} u g$, $\forall g \in G_{0}$. We can now proceed to show that given such $u$ one can obtain a $\sigma$, and vice versa.

$\Rightarrow$ A dressing field as above induce the trivialization:

$$
\begin{aligned}
\phi_{u}: \mathcal{P} & \rightarrow \mathcal{P} / K \times K \\
p & \mapsto\left(p u(p), u(p)^{-1}\right) \\
p k & \mapsto\left(p k u(p k), u(p k)^{-1}\right)=\left(p u(p), u(p)^{-1} k\right) \\
p g & \mapsto\left(p g u(p g), u(p g)^{-1}\right)=\left(p u(p) g, g^{-1} u(p)^{-1} g\right) .
\end{aligned}
$$

Now, a trivialization allows to define a global section and vice versa. Here we define $\sigma_{u}: \mathcal{P} / K \rightarrow \mathcal{P}$ by:

$$
\begin{aligned}
\phi_{\sigma_{u}}^{-1}: \mathcal{P} / K \times K & \rightarrow \mathcal{P} \\
\left(p u(p), u(p)^{-1}\right) & \mapsto p u(p) \cdot u(p)^{-1}=: \sigma_{u}(p u(p)) \\
\left(p k u(p k), u(p k)^{-1}\right)=\left(p u(p), u(p)^{-1} k\right) & \mapsto p k=: \sigma_{u}(p u(p)) k \\
\left(p g u(p g), u(p g)^{-1}\right)=\left(p u(p) g, g^{-1} u(p)^{-1} g\right) & \mapsto \sigma_{u}(p u(p) g)=p g=\sigma_{u}(p u(p)) g .
\end{aligned}
$$

Thus we indeed obtain the $G_{0}$-equivariance property $R_{g}^{*} \sigma_{u}=R_{g} \circ \sigma_{u}$.

$\Leftarrow$ Suppose we have a $G_{0}$-equivariant global section $\sigma: \mathcal{P} / K \rightarrow \mathcal{P}$, it induces the corresponding trivialization:

$$
\begin{aligned}
\phi_{\sigma}^{-1}: \mathcal{P} / K \times K & \rightarrow \mathcal{P} \\
(x, e) & \mapsto \sigma(x) \\
(x, k) & \mapsto \sigma(x) k \\
(x g, e)=\left(x g, g^{-1} e g\right) & \mapsto \sigma(x g)=\sigma(x g) g^{-1} e g=\sigma(x) g \\
\left(x g, g^{-1} k g\right) & \mapsto \sigma(x g) g^{-1} k g=\sigma(x) k g .
\end{aligned}
$$

In the last two equalities of the last two rows, the equivariance property of $\sigma$ is used. A $K$-dressing field is thus defined via:

$$
\begin{aligned}
u_{\sigma}: \mathcal{P} & \rightarrow K, \\
\sigma(x) & \mapsto u_{\sigma}(\sigma(x))=e^{-1} \\
\sigma(x) k & \mapsto u_{\sigma}(\sigma(x) k)=k^{-1}=k^{-1} e^{-1}=k^{-1} u_{\sigma}(\sigma(x)) \\
\sigma(x) g & \mapsto u_{\sigma}(\sigma(x) g)=e^{-1}=g^{-1} e^{-1} g=g^{-1} u_{\sigma}(\sigma(x)) g \\
\sigma(x) k g & \mapsto u_{\sigma}(\sigma(x) k g)=g^{-1} k^{-1} g=g^{-1} u_{\sigma}(\sigma(x) k) g .
\end{aligned}
$$


Thus we obtain indeed the defining property of a $K$-dressing fied, $R_{k}^{*} u_{\sigma}=k^{-1} u_{\sigma}$, and its residual $G_{0}$-equivariance $R_{g}^{*} u_{\sigma}=g^{-1} u_{\sigma} g$, as in Proposition 2 .

Notice however the following fact. Given forms $\alpha_{i}$ on $\mathcal{P}$ which is endowed with a Weyl structure $\sigma$, they can be pulled-bak on $\mathcal{P} / K \subset \mathcal{P}$ as $a_{i}:=\sigma^{*} \alpha_{i}$. But as a section, $\sigma$ supports a $\mathcal{K}$-action: given $\Psi \in \operatorname{Aut}_{v}^{K}(\mathcal{P})$ acting as $\Psi(p)=p k(p)$ with $k \in \mathcal{K}$, another Weyl structure is given by $\hat{\sigma}(x)=\Psi(\sigma(x))=\sigma(x) k(\sigma(x))$. Therefore, the $a_{i}$ 's support an action by the gauge group $\mathcal{K}$ defined by: $a_{i}^{k}:=\Psi^{*} a_{i}=\hat{\sigma}^{*} \alpha_{i} \neq a_{i}$.

The composite fields defined in Proposition 1 can be obtained as pullbacks via the map $f_{u}: \mathcal{P} \rightarrow \mathcal{P} / K \subset \mathcal{P}$ defined by $f_{u}(p):=p u(p)$. By construction, the latter satisfies: $f_{u} \circ \Psi(p)=f_{u}(p k(p))=p k(p) u(p k(p))=p k(p) k(p)^{-1} u(p)=p u(p)=f_{u}(p)$. Therefore, the composite fields $\alpha_{i}^{u}:=f_{u}^{*} \alpha_{i}$ are by construction $\mathcal{K}$-gauge invariant: $\left(\alpha_{i}^{u}\right)^{k}:=\Psi^{*}\left(\alpha_{i}^{u}\right)=$ $\Psi^{*} f_{u}^{*} \alpha_{i}=\left(f_{u} \circ \Psi\right)^{*} \alpha_{i}=f_{u}^{*} \alpha_{i}=: \alpha_{i}^{u}$.

So, despite the fact that in the specific case of a $|1|$-graded parabolic geometry $\mathcal{P}$, a dressing field with particular residual $G_{0}$-equivariance is equivalent to a Weyl structure, what is achieved by the former is distinct from what is achieved via the latter. This highlights again the general feature that the DFM is a non-dynamical gauge reduction scheme, which is the crucial point of the argument presented in this paper regarding the physical (ir)relevance of (artificial/fake) gauge symmetries - Weyl symmetry in the case at hand.

Open Access. This article is distributed under the terms of the Creative Commons Attribution License (CC-BY 4.0), which permits any use, distribution and reproduction in any medium, provided the original author(s) and source are credited.

\section{References}

[1] K. Hinterbichler and J. Khoury, The Pseudo-Conformal Universe: Scale Invariance from Spontaneous Breaking of Conformal Symmetry, JCAP 04 (2012) 023 [arXiv:1106.1428] [INSPIRE].

[2] I. Bars, P. Steinhardt and N. Turok, Local Conformal Symmetry in Physics and Cosmology, Phys. Rev. D 89 (2014) 043515 [arXiv:1307.1848] [InSPIRE].

[3] G. 't Hooft, Local conformal symmetry in black holes, standard model and quantum gravity, Int. J. Mod. Phys. D 26 (2016) 1730006 [inSPIRE].

[4] M. Atiyah, M. Dunajski and L. Mason, Twistor theory at fifty: from contour integrals to twistor strings, Proc. Roy. Soc. Lond. A 473 (2017) 20170530 [arXiv:1704.07464] [INSPIRE]. [http://rspa.royalsocietypublishing.org/content/473/2206/20170530.full.pdf].

[5] S. Speziale, Loop quantum gravity, twistors and some perspectives on the problem of time, EPJ Web Conf. 71 (2014) 00123 [arXiv: 1404.4167] [InSPIRE].

[6] T. Bailey, M. Eastwood and A. Gover, Thomas's structure bundle for conformal, projective and related structures, Rocky Mt. J. Math. 24 (1994) 1191.

[7] A.R. Gover, A. Shaukat and A. Waldron, Tractors, Mass and Weyl Invariance, Nucl. Phys. B $812(2009) 424$ [arXiv:0810.2867] [INSPIRE]. 
[8] R.W. Sharpe, Differential Geometry: Cartan's Generalization of Klein's Erlangen Program, vol. 166 of Graduate text in Mathematics, Springer, (1996).

[9] A. Cap and J. Slovak, Parabolic Geometries I: Background and General Theory, vol. 1 of Mathematical Surveys and Monographs, American Mathematical Society, (2009).

[10] J. Attard and S. Lazzarini, A note on Weyl invariance in gravity and the Wess-Zumino functional, Nucl. Phys. B 912 (2016) 289 [arXiv:1607.06326] [INSPIRE].

[11] R. Jackiw and S.-Y. Pi, Fake Conformal Symmetry in Conformal Cosmological Models, Phys. Rev. D 91 (2015) 067501 [arXiv:1407.8545] [INSPIRE].

[12] J. Attard, J. François, S. Lazzarini and T. Masson, The dressing field method of gauge symmetry reduction, a review with examples, in Foundations of Mathematics and Physics one Century After Hilbert: New Perspectives, Springer, (2018), [arXiv:1702.02753] [INSPIRE].

[13] J. François, Artificial vs Substantial Gauge Symmetries: a Criterion and an Application to the Electroweak Model, arXiv:1801.00678 [INSPIRE].

[14] J. Attard and J. François, Tractors and Twistors from conformal Cartan geometry: a gauge theoretic approach I. Tractors, arXiv:1609.07307 [INSPIRE].

[15] S.N. Curry and A.R. Gover, An Introduction to Conformal Geometry and Tractor Calculus, with a view to Applications in General Relativity, pp. 86-170, Lond. Math. Soc. Lect. Note Ser., Cambridge University Press, (2018).

[16] R. Penrose and W. Rindler, Spinors and Space-Time, vol. 1., Cambridge University Press, (1984).

[17] R. Penrose and W. Rindler, Spinors and Space-Time, vol. 2., Cambridge University Press, (1986).

[18] J. Attard and J. François, Tractors and Twistors from conformal Cartan geometry: a gauge theoretic approach II. Twistors, Class. Quant. Grav. 34 (2017) 085004 [arXiv:1611.03891] [INSPIRE].

[19] M.D. Pollock, On the Dirac equation in curved space-time, Acta Phys. Polon. B 41 (2010) 1827 [INSPIRE].

[20] V. Bargmann, Bemerkungen zur allgemein-relativistischen fassung der quantentheorie, Sitzungsber. Preuss. Akad. Wiss. 1932 (1932), no. XXIV 346.

[21] J. François, Reduction of gauge symmetries: a new geometrical approach, Thesis, Aix-Marseille Université, France, (2014).

[22] J. Collins, A. Perez and D. Sudarsky, Lorentz invariance violation and its role in Quantum Gravity phenomenology, Cambridge University Press, (2009), pp. 528-547.

[23] D. Colladay and V.A. Kostelecký, Lorentz violating extension of the standard model, Phys. Rev. D 58 (1998) 116002 [hep-ph/9809521] [INSPIRE].

[24] D. Mattingly, Modern tests of Lorentz invariance, Living Rev. Rel. 8 (2005) 5 [gr-qc/0502097] [INSPIRE].

[25] O.W. Greenberg, CPT violation implies violation of Lorentz invariance, Phys. Rev. Lett. 89 (2002) 231602 [hep-ph/0201258] [INSPIRE].

[26] O. Bertolami, D. Colladay, V.A. Kostelecký and R. Potting, CPT violation and baryogenesis, Phys. Lett. B 395 (1997) 178 [hep-ph/9612437] [INSPIRE]. 
[27] S.M. Carroll and J. Shu, Models of baryogenesis via spontaneous Lorentz violation, Phys. Rev. D 73 (2006) 103515 [hep-ph/0510081] [INSPIRE].

[28] M. Campigotto and L. Fatibene, Gauge natural formulation of conformal gravity, Annals Phys. 354 (2015) 328 [arXiv: 1404.0898] [InSPIRE].

[29] J.B. Pitts, General Covariance, Artificial Gauge Freedom and Empirical Equivalence. Ph.D. Thesis, Graduate School of the University of Notre Dame, France, (2008).

[30] J.B. Pitts, Empirical Equivalence, Artificial Gauge Freedom and a Generalized Kretschmann Objection, arXiv:0911.5400 [INSPIRE].

[31] M.P. Hertzberg, Inflation, Symmetry and B-Modes, Phys. Lett. B 745 (2015) 118 [arXiv: 1403.5253] [INSPIRE].

[32] G. 't Hooft, Local conformal symmetry: The missing symmetry component for space and time, Int. J. Mod. Phys. D 24 (2015) 1543001 [inSPIRE].

[33] W. Pauli, Über die Formulierung der Naturgesetze mit fünf homogenen Koordinaten. Teil II: Die Diracschen Gleichungen für die Materiewellen, Annalen Phys. 18 (1933) 337.

[34] R.U. Sexl and H.K. Urbantke, Relativity, Groups, Particles: Special Relativity and Relativistic Symmetry in Field and Particle Physics, Springer-Verlag Wien, (2001).

[35] M. Arminjon and F. Reifler, Basic quantum mechanics for three Dirac equations in a curved spacetime, Braz. J. Phys. 40 (2010) 242. 